\title{
Effect of the number of uninvolved nodes on survival in early breast cancer
}

\author{
VINCENT VINH-HUNG ${ }^{1}$, GÁBOR CSERNI ${ }^{2}$, TOMASZ BURZYKOWSKI ${ }^{3}$, \\ JAN VAN DE STEENE ${ }^{1}$, MIA VOORDECKERS ${ }^{1}$ and GUY STORME ${ }^{1}$ \\ ${ }^{1}$ Department of Radiotherapy, Oncologisch Centrum, AZ-VUB, 101 Laarbeeklaan, B-1090 Jette, Belgium; \\ ${ }^{2}$ Surgical Pathology, Bács-Kiskun County Hospital, Nyiri ut 38, H-6000 Kecskemét, Hungary; \\ ${ }^{3}$ Center for Statistics, Limburgs Universitair Centrum, Universitaire Campus Building D, B-3590 Diepenbeek, Belgium
}

Received July 22, 2002; Accepted September 11, 2002

\begin{abstract}
A high number of uninvolved axillary nodes was found by some authors to be associated with poor survival in node-negative breast cancer. We searched for confirmation and extend the investigation to node-positive cases, using population data from the SEER Program. Patients selected were women aged 40-69 years, diagnosed 1988-1997, T1-T2 breast cancer, undergoing axillary dissection with 4-35 nodes examined. Survivals were estimated by the product-limit method and were computed on pooled data. Results in nodenegative patients $(n=37,519)$ showed a 5 -year overall survival from $92 \%$ (95\% confidence interval: $88-95 \%$ ) with 4 uninvolved nodes, to $93 \%$ (87-98\%) with 34 uninvolved nodes. In node-positive patients $(n=16,978)$, the 5-year survival increased from $50 \%$ (44-56\%) with 0 uninvolved nodes, to $91 \%(82-100 \%)$ with 30 uninvolved nodes. Survival graphs indicated an improvement or a plateau with higher number of uninvolved nodes. The graphs also suggested that the ratio of involved and uninvolved nodes might be correlated with survival. We conclude that there was no evidence of poor outcome associated with a high number of uninvolved nodes. The incidental finding that ratio-based characterization of node involvement might be a prognostic factor will be further investigated.
\end{abstract}

Correspondence to: Dr V. Vinh-Hung, Oncologisch Centrum, AZ-VUB, 101 Laarbeeklaan, B-1090 Jette, Belgium

E-mail: conrvhgv@az.vub.ac.be

Abbreviations: N0 node-negative, negative nodal status; $\mathrm{N}^{+}$nodepositive, positive nodal status; nneg, number of uninvolved nodes; npos, number of involved nodes; nex, number of examined nodes; SEER, Surveillance, Epidemiology, and End Results; VEGF, vascular endothelial growth factor

Key words: breast neoplasms, axilla, lymph nodes, dissection, SEER program, survival analysis, diagnostic techniques, surgical, prognosis, neoplasm staging, models, biological, ratio-based staging

\section{Introduction}

Tumor draining lymph nodes can undergo hyperplasia, resulting in increases in the number and size of detectable lymph nodes. A high number of tumor free axillary lymph nodes might thus represent the effect of lymphangiogenic and lymph node development factors playing a role in tumor metastasis (1). In support of that hypothesis, Camp et al reported that axillary dissection yielding more than 20 nodes in node-negative breast cancer was associated with a poor survival (1) when compared with node-negative breast cancer patients having less than 20 nodes examined.

The Surveillance, Epidemiology, and End Results (SEER) provides detailed data on nodal extent for cases diagnosed since 1988 (2). The objective of the present study is to examine the pattern of survival, whether or not there is supportive evidence in the SEER registries data that high numbers of uninvolved nodes (nneg) are indicators of poor outcome.

If lymphogenic factors are involved in node-negative (N0) breast cancer, then it should be expected that these factors are also involved in node-positive $\left(\mathrm{N}^{+}\right)$cases. Therefore, consistency requires that the role of nneg should be examined in both $\mathrm{NO}$ and $\mathrm{N}^{+}$cases, taking into account the number of positive nodes (npos).

Outline of this study and results of a multivariate analysis by proportional hazard models have been reported previously (3). The mortality hazard ratio associated with the number of uninvolved nodes was 0.990 (95\% confidence interval: $0.983-0.996)$ in $\mathrm{N} 0$, and $0.970(0.963-0.976)$ in $\mathrm{N}^{+}$, i.e. $1 \%$ risk reduction and $3 \%$ risk reduction per uninvolved node, respectively. These results argued against the role of uninvolved nodes as indicators of poor prognosis. The present report will focus on the visual display of data. We believe that accurate and comprehensive visual presentation of data that identifies relationships is important (4).

\section{Materials and methods}

Patients selected from the SEER database (2) were women aged 40-69 years with non-inflammatory invasive carcinoma, diagnosed between 1988 and 1997, with primary tumor of $\leq 50 \mathrm{~mm}$ and confined to breast, without previous diagnosis 
of cancer, without identified internal mammary node involvement or distant metastases, treated by partial or total mastectomy, with or without post-surgery radiation, with axillary dissection resulting in the examination of 4 to 35 nodes. Follow-up cut-off date was December 31, 1997.

Survival estimates were calculated using the product-limit method (5). Event was defined as death from any cause. Cases were analyzed by: a) number of uninvolved nodes (nneg); b) number of involved nodes (npos); c) nodal status: $\mathrm{N} 0$, node negative (npos $=0$ ) and $\mathrm{N}^{+}$, one or more nodes involved (npos $>0$ ).

To simplify graphical presentations, it was decided to use survival estimates at a single time-point. All estimated survival curves were inspected to ascertain that there was no unusual change of hazards over the time range. The screening verified that early survival estimates were reasonably predictive of late survival estimates. Considering the trade-off between the choice of the time-point and precision of estimates, single 5-year survival estimates were selected as indicators of survival to be used in the graphical representations.

Different representations with different levels of pooling data were used: a) univariate representations of 5-year survival used the estimates obtained by the product-limit method without pooling (Figs. 1 and 2); b) panel representation of survival estimates in function of nneg for different values of npos pooled data from patients with neighboring values of nneg (Fig. 3). That is, for each category of values of npos (npos $=1,2,3,4-5,6-9,10-35)$, survival estimates for any given value of nneg, nneg ${ }_{0}$ say, were computed on the subset of records for which nneg ranged between $\left(\right.$ nneg $\left._{0}-3\right)$ and $\left(\right.$ nneg $\left._{0}+3\right)$. The range was truncated at the extremes of the selection range. The pooling was used to compensate for a small number patients for some combinations of nneg and npos; c) three-dimensional representations, either as an elevation map (Fig. 4) or as a contour plot (Fig. 5), computed the 5-year survival estimates in function of npos and nneg. For any given pair of values $\left(\mathrm{nneg}_{0}, \mathrm{npos}_{0}\right), 5$-year survival was estimated on the subset of patients for whom nneg ranged between $\left(\right.$ nneg $\left._{0}-2\right)$ and $\left(\right.$ nneg $\left._{0}+2\right)$ and for whom npos ranged between $\left(\right.$ npos $\left._{0}-2\right)$ and $\left(\operatorname{npos}_{0}+2\right)$. The ranges were truncated at the extremes of the selection range. The pooling was used to compensate for a small number of patients for some pairs of $\left(\right.$ nneg $\left._{0}, \operatorname{npos}_{0}\right)$. The elevation map in Fig. 4 was further smoothed by using Shepard's inverse distance method (6) to obtain a continuous surface.

Computations of survival estimates were performed using JMP 4 (SAS Institute Inc., Cary, NC, USA). Plots and implementation of the inverse distance method were obtained using SigmaPlot 2001 (SPSS Science, Chicago, IL, USA).

\section{Results}

Available data. There were 236 patients with $<4$ nodes examined (nex) and 608 patients with 35 to $>90$ nex. These cases were excluded because of distribution gaps and because of the too small numbers in view of the intended methods. A total of 54,497 records matching selection criteria were retained and represent the study population, 37,519 patients N0,16,978 patients $\mathrm{N}^{+}$. Median follow-up of patients still alive at cut-off date was 50 months (mean 53).

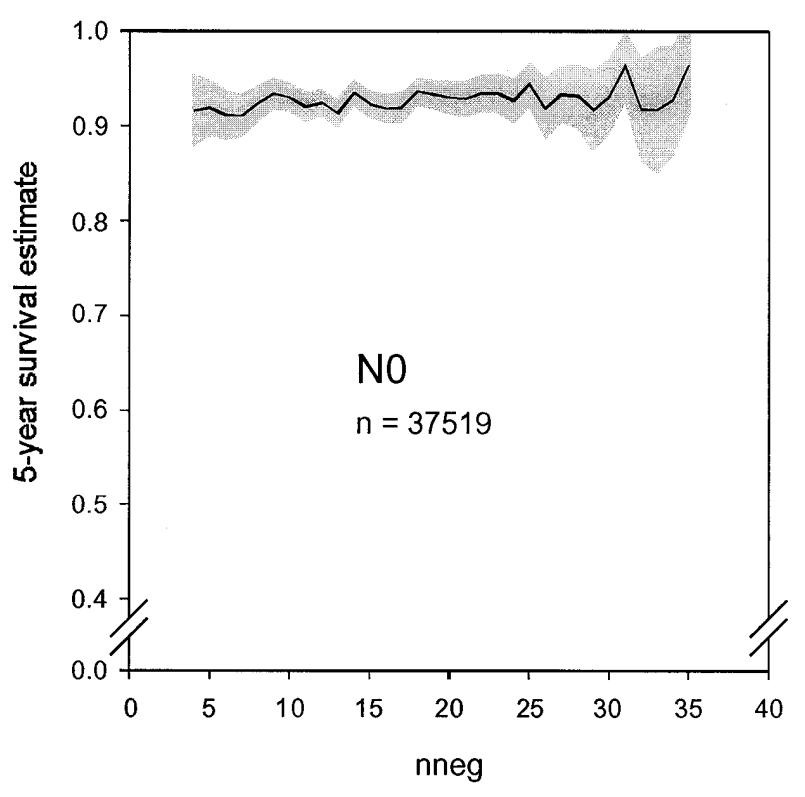

Figure 1. Survival in node-negative T1-T2 breast cancer in function of the number of uninvolved nodes. Unsmoothed 5-year overall survival estimates in node-negative patients. Shaded area is $95 \%$ confidence interval.

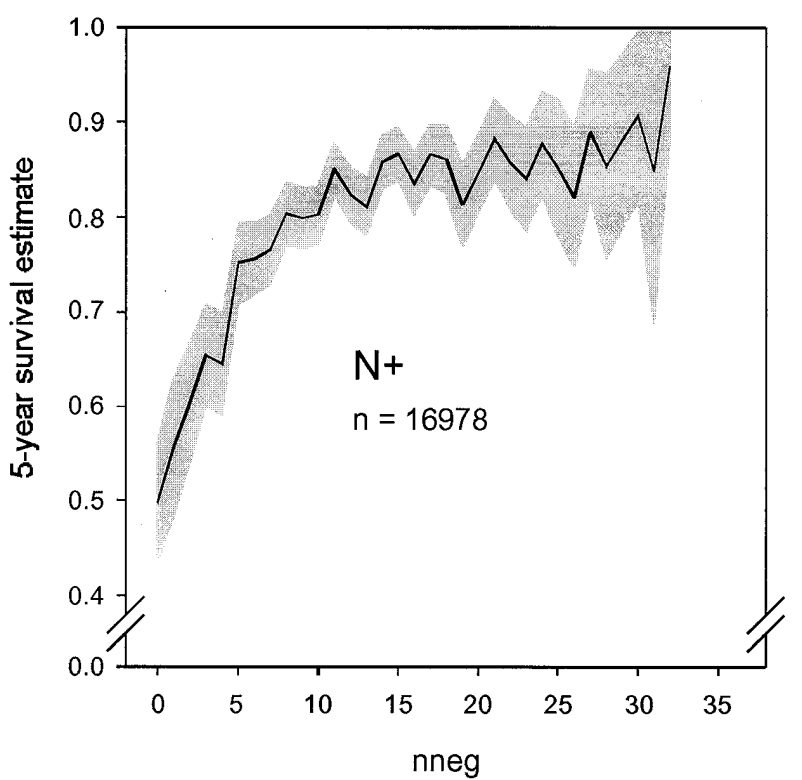

Figure 2. Survival in node-positive T1-T2 breast cancer in function of the number of uninvolved nodes. Unsmoothed 5-year overall survival estimates in node-positive patients. Shaded area is $95 \%$ confidence interval.

Median follow-up of patients who died was 40 months (mean 45). Detailed characteristics of patients have been presented elsewhere (7), a summary is shown in Table I.

Survival by nodal status. Survival appeared practically constant in N0 patients, there was no decrease of survival with higher values of nneg (Fig. 1). Note that there are no estimates for very low values of nneg since the selection of patients specified the examination of at least four nodes. A steep improvement 


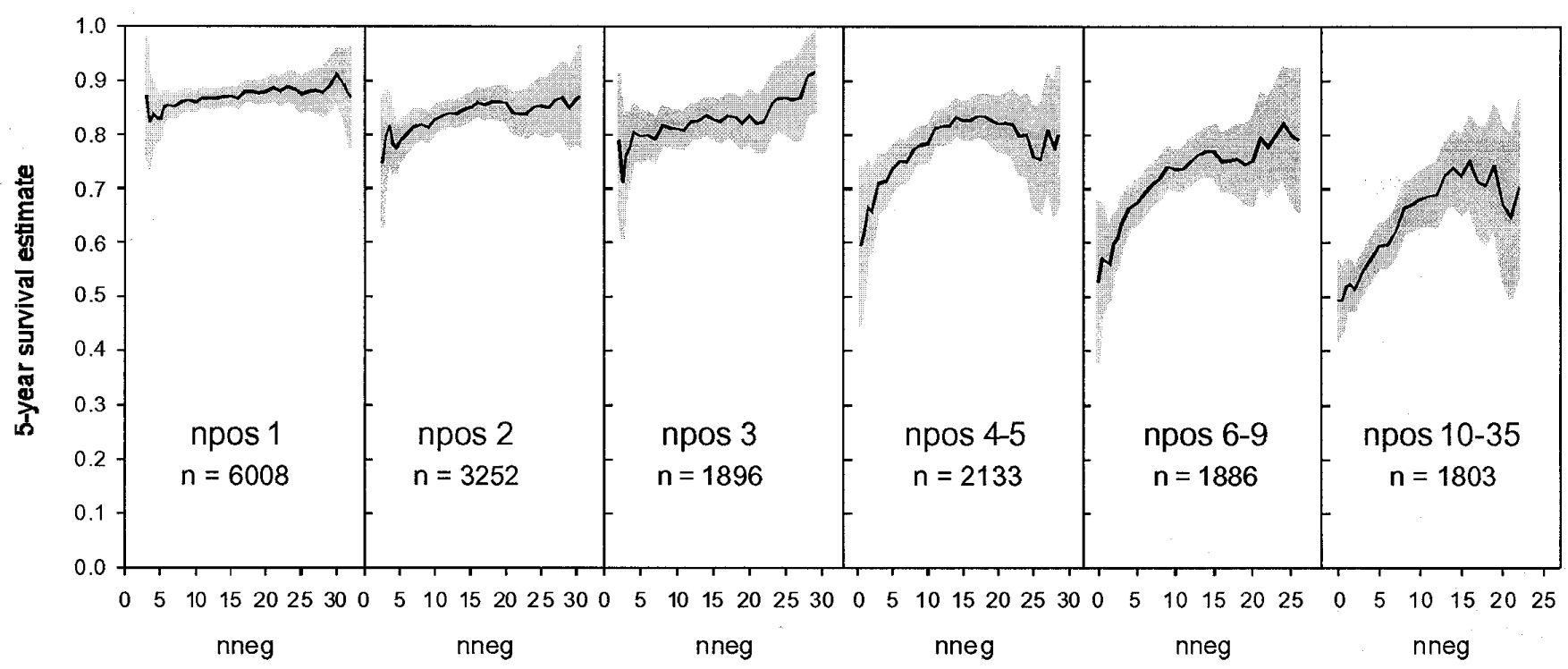

Figure 3. Panel display of survival in T1-T2 breast cancer in function of the numbers of uninvolved nodes and of involved nodes. Five-year overall survival estimates in function of the pooled number of uninvolved nodes (nneg), when the number of involved nodes (npos) is $1,2,3,4$ to 5,6 to 9 and 10 to 35 . Shaded area is $95 \%$ confidence interval.

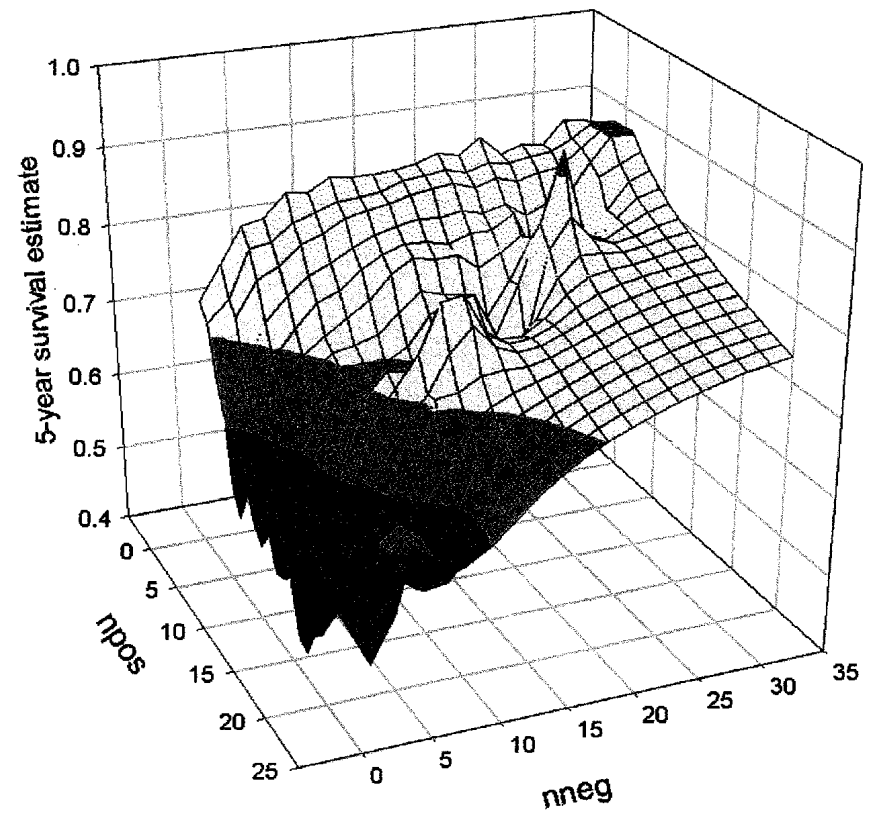

Figure 4. Bivariate display of survival in T1-T2 breast cancer in function of the numbers of uninvolved nodes and of involved nodes. The surface beyond 35 nodes examined was extrapolated. Survival deteriorates when the number of involved nodes increases. Survival improves when the number of uninvolved nodes increases, steeply at lower ranges, minutely at higher ranges. The information content is similar to Fig. 3. Confidence intervals are not displayed here, but the accidental-type of adjacent dips and peaks are better highlighted.

of survival followed by a plateau was observed in $\mathrm{N}^{+}$patients with increasing nneg (Fig. 2).

Detailed pattern by number of nodes. Stratification in function of npos showed that within each npos stratum, survival improved with increasing nneg (Fig. 3).

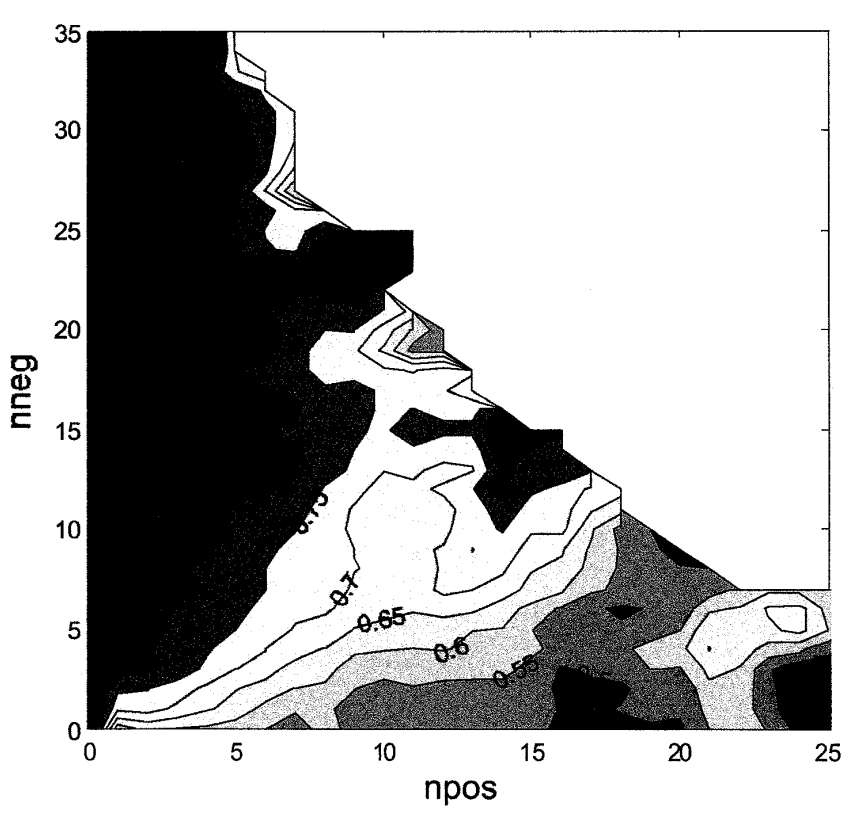

Figure 5. Conjoint effect of the numbers of uninvolved nodes and of involved nodes on survival in T1-T2 breast cancer. Part of the contour plot was partially filled at the corners by padding. Similar ratios of involved/uninvolved nodes are associated with similar survival, e.g. 8 npos/10 nneg has approximately the same $75 \%$ (contour line 0.75 ) 5-year survival chance as 4 npos $/ 5$ nneg.

The survival in function of npos and nneg displayed as a 3D smoothed plot showed no decrease of survival with large values of nneg (Fig. 4).

The survival in function of npos and nneg was further displayed as a filled contour plot in which 5-year survivals of $50-60 \%$ were coded blue, $70 \%$ green yellow, $80 \%$ orange, $90 \%$ dark red (Fig. 5). For any given npos, survival increases when nneg increases (changes of color vertically). The survival increase is not uniform, it plateaus with large nneg at small 
Table I. Summary of patient characteristics.

\begin{tabular}{|c|c|c|c|c|c|c|}
\hline \multirow[b]{2}{*}{ Characteristic } & \multicolumn{3}{|c|}{ No } & \multicolumn{3}{|c|}{$\mathrm{N}^{+}$} \\
\hline & $\begin{array}{c}\text { Total } \\
\mathrm{n}=37,519\end{array}$ & $\begin{array}{l}n e x>20 \\
n=8,514\end{array}$ & $\begin{array}{c}\text { nex }<20 \\
n=29,005\end{array}$ & $\begin{array}{c}\text { Total } \\
\mathrm{n}=16,978\end{array}$ & $\begin{array}{l}\text { nex }>20 \\
n=4,580\end{array}$ & $\begin{array}{c}\text { nex }<20 \\
n=12,398\end{array}$ \\
\hline Age $>50$ years & 27,326 & 6,015 & 21,311 & 11,137 & 2,917 & 8,220 \\
\hline Tumor T2-stage & 8,643 & 2,080 & 6,563 & 8,287 & 2,327 & 5,960 \\
\hline Histologic grade 3 & 9,091 & 2,174 & 6,917 & 5,900 & 1,633 & 4,267 \\
\hline ER negative (1990+) & 6,074 & 1,414 & 4,660 & 3,014 & 822 & 2,192 \\
\hline PR negative (1990+) & 7,710 & 1,726 & 5,984 & 3,781 & 1,019 & 2,762 \\
\hline Radiation & 17,042 & 3,606 & 13,436 & 6,363 & 1,699 & 4,664 \\
\hline Dead of breast cancer & 1,320 & 320 & 1,000 & 2,355 & 648 & 1,707 \\
\hline Dead of other cause & 1,329 & 299 & 1,030 & 737 & 208 & 529 \\
\hline
\end{tabular}

N0, node-negative; $\mathrm{N}^{+}$, node-positive; nex, number of examined nodes.

npos (color does not change anymore vertically beyond nneg 5-10 at npos 0-4). For any given nneg, survival decreases when npos increases (changes of color horizontally). The survival levels with large npos at small nneg (color does not change anymore horizontally beyond npos 8-15 at nneg 0-3). For any given pair of (nneg, npos), survival is approximately constant if the ratio nneg/npos remains the same (color bands radially oriented).

\section{Discussion}

No unfavorable effect of the number of uninvolved nodes. The number of tumor free lymph nodes was unexpectedly found to be an independent predictor of aggressive disease in cases of lymph node-negative breast carcinoma in a cohort of 290 node-negative patients treated in 1983-1993 (1). In a more recent study based on a larger study population of 911 patients treated in 1985-1993, Moorman et al failed to confirm the finding (8). They noted that a previous study on 464 T1N0 patients found significantly better survival among those with more lymph nodes examined (9). It was concluded that the overall lack of consistency in the findings of the three studies suggests that it is unlikely that the number of lymph nodes examined could be a reliable prognostic indicator in nodenegative breast carcinoma (8).

Our current analysis finds no worse survival for nodenegative patients with more nodes removed. The results are in keeping with the largest study on this matter known to us (8).

Extending the analysis on node-positive patients found no adverse survival associated with large number of tumor free nodes either. Two dips in survival were observed but the very large confidence intervals (Fig. 3) and the adjacency with two large peaks (Fig. 4) were more suggestive of data fluctuation than of any reliable effect.

Biological contradiction? Because the larger number of nodes examined would suggest a more reliable nodal staging of nodenegative breast carcinomas, the finding of a worse prognosis with more negative nodes examined in a cohort of nodenegative patients required some explanation. The rationale discussed by Camp et al which originally hypothesized that a high number of tumor free nodes would be related to poor prognosis, was that increases in the number of nodes might be related to hyperplasia of microscopic nodes exposed to tumor cytokines, or might be related to lymphangiogenic factors secreted by tumors (1).

On one hand, our results show that the poor prognosis of a high number of tumor free nodes is not substantiated. On the other hand there is strong recent experimental supportive evidence for the role of lymphangiogenic factors. Next we consider that experimental evidence and whether or not it is in contradiction with our results.

Pronounced lymphangiogenesis was found within human metastatic breast tumors transplanted in nude mice (10). Overexpression of vascular endothelial growth factor (VEGF)-C, an analog of VEGF, resulted in enlargement of peritumoral lymphatic vessels, in increased intratumoral lymphangiogenesis, in enhanced rates of lymphatic metastasis and in a significant increase of lung metastases (10).

Cell lines that express VEGF-D, another structural analog of VEGF-C, were shown to induce high vascularization and formation of lymphatics within tumors, whereas VEGF induced tumor angiogenesis but not lymphangiogenesis (11). Importantly, VEGF-D was found to promote lymphatic spread of tumors, whereas VEGF did not (11).

It follows from these experimental findings that, if lymphangiogenesis occurs, then lymphatic metastasis is also enhanced, and consequently it would be unlikely to find exclusively uninvolved nodes. But, if lymphangiogenesis occurs, and yet no or few nodes are involved, then it would imply something else happened, e.g. host protective mechanism against lymphatic metastasis (12) or the lack of a further unspecified metastasis promoting factor.

The biological implication is thus opposite to the hypothesis of Camp et al (1) which stated poorer survival, but is consistent with the present findings that a large number of uninvolved nodes is not an indicator of poor survival. 
It should be emphasized that Fisher et al attributed a particular significance to node-negativity as indicating protective mechanism (12). In a most recent communication, De Paola et al reported for node-negative breast cancer an apparently paradoxical survival trend in favor of patients with high VEGF-expressing tumors, although high VEGF expression was consistently associated with high microvessel density, a known factor of poor prognosis (13). Even though the number of nodes was not investigated in that report, the convergence with the deduction of Fisher et al and with the implication of the animal models is remarkable. The possible link between VEGFs and the number of nodes warrants further studies.

Incidental findings on axillary node dissection. Axillary node dissection is the subject of a debate with many facets. The present report does not address but overlaps with the debate. The number of negative nodes is contingent on the number of nodes examined and on the number of positive nodes, nneg = nex - npos, thus is contingent on extent of axillary dissection. Since the analysis explicitly took into account npos, nex is fully defined, some discussion on the extent of node dissection is warranted.

The practically flat curve in NO suggests that N0 patients derived little substantial survival benefit from extensive node examination, from $92 \%$ 5-year survival with five nodes examined to less than $94 \%$ with 35 nodes examined (Fig. 1). This is an apparently intriguing counter-intuitive result. If few nodes have been removed, should not there have been a high risk of involved nodes being left behind, and, if so, does not that mean that involved nodes had only a negligible impact on survival, in contradiction with the obvious worsened survival when nodes are involved (Figs. 3-5)?

Randomized trials that have directly compared limited axillary dissection or sampling with more extensive dissection yielded similar rates of nodal status distribution (14-18) and similar survival or mortality between the two arms $(14,17,18)$. These trials showed comparable efficiency of limited axillary exploration, with a condition highlighted by Steele et al: '... it is important to emphasize that it is the surgeon who must take the responsibility for identifying nodes and in our unit a satisfactory sampling procedure now requires the identification of four nodes fixed for histology by the operating surgeon' (15).

In a prospective clinical study by the Uppsala, Örebro and Karolinska Institutes, 415 consecutive patients were operated in the axilla with a sequential five-node biopsy followed at the same operation by a further dissection of level I-II of the axilla in order to evaluate the accuracy of the five-node biopsy (19). A total of 149 patients were node-positive. The first four nodes identified $143(96 \%)$ of these cases. Five nodes identified $145(97.3 \%)$ cases.

In an experimental pathology study of 499 axillary clearance specimens at the Bács-Kiskun Hospital, Cserni compared assessment of the first three, four, five, or six largest/firmest nodes against assessment of all axillary nodes (20). The first three, four, five and six nodes identified $94-96 \%$, 97-98\%, 98-99\% and 99\% of node-positive cases, respectively.

The above mentioned randomized trials showed that efficiency of limited axillary dissection or sampling and more extensive dissection were comparable. The Uppsala-Orebro prospective clinical study and the Bács-Kiskun pathology experiment independently suggest an explanation: axillary sampling or biopsy is not a blind procedure in which some arbitrary amount of fat is removed by the surgeon from which nodes are randomly picked out by the pathologist. Another of our earlier studies has documented that qualitative features of the nodes (larger size, firm consistency) make nodes most likely to be involved easier to identify, and the removal of further small nodes does not really help further (21). So retrieval of the nodes is not a random process, some nodes are more likely to be found, and these seem to overlap with those that are more likely to have metastases (22). The quite flat survival curve of Fig. 1 is consistent with the nonrandomness of axillary examination. It suggests that in the practice covered by the SEER registries, in the selected population of patients, the surgeon and the pathologist most probably correctly identified the node-positive cases, thus the remaining node-negative cases, whether based on 5 nodes, or 10 , or 25 , or 35 , possibly had a low false negative rate reflected by the small impact on survival. In essence, the present observation is a rediscovery of findings made by Fisher et al more than 20 years ago: 'The prognosis of patients with carcinoma of the breast was essentially the same when 5 or 10 nodes were removed and were reported to be negative as when 25 or 30 nodes were found to be free of tumor' (12).

This review of the literature and the present results appear to suggest that the classification of patients as node-negative might be based on a limited axillary dissection, e.g. node sampling or sentinel node biopsy $(23,24)$. However, most of the published results come from single institutions, while results from the SEER come from more heterogeneous practices and consequently should not be interpreted as indicating four or five nodes as a prognostic cut-off. The formal search of a cut-off number of nodes that takes into account the heterogeneity of the data is more complex and will be presented in a forthcoming report. Furthermore, another incidental finding that has not yet been commented on is the relation between survival and the ratio of involved and uninvolved nodes. A few studies have investigated ratiobased staging in gastric cancer $(25,26)$. Fig. 5 suggests that the ratio might also be an important prognostic factor in breast cancer. That possibility will be considered with the investigation of node cut-off.

In conclusion, the present detailed descriptive analysis of the SEER population data on stage I-II breast cancer finds no consistent evidence of any unfavorable outcome associated with high number of uninvolved axillary nodes, whatever the nodal status of the patients.

Incidental findings suggest that the ratio of involved and uninvolved nodes might be an important prognostic factor. The ratio will be considered in a model-based study of the node prognostic cut-off.

\section{References}

1. Camp RL, Rimm EB and Rimm DL: A high number of tumor free axillary lymph nodes from patients with lymph node negative breast carcinoma is associated with poor outcome. Cancer 88: 108-113, 2000. 
2. Surveillance, Epidemiology, and End Results (SEER) Program Public-Use Data (1973-1997), National Cancer Institute, DCCPS, Cancer Surveillance Research Program, Cancer Statistics Branch. National Cancer Institute, Bethesda, MD, 2000 (http://seer. cancer.gov).

3. Vinh-Hung V, Cserni G, Burzykowski T, van de Steene J and Storme G: Is a high number of uninvolved nodes in early breast cancer an indicator of poor outcome? Eur J Cancer 37 (Suppl 6): S187, 2001.

4. Vinh-Hung V and Storme G: Visual display of epidemiological time series (age, period, cohort data). Bulletin of the International Statistical Institute, 52nd session, LVIII Contributed Papers Book 3. International Statistical Institute, Helsinki, pp447-448, 1999 (http://www.stat.fi/isi99/proceedings/arkisto/ contributed/91.html).

5. Kaplan E and Meier P: Non-parametric estimation from incomplete observations. J Am Stat Assoc 53: 457-481, 1958.

6. Shepard D: A two-dimensional interpolation function for irregularly spaced data. Brandon/Systems Press, Princeton, NJ, pp517-524, 1968.

7. Vinh-Hung V, Burzykowski T, van de Steene J, Storme G and Soete G: Post-surgery radiation in early breast cancer: survival analysis of registry data. Radiother Oncol 64: 281-290, 2002.

8. Moorman PG, Hamza A, Marks JR and Olson JA: Prognostic significance of the number of lymph nodes examined in patients with lymph node-negative breast carcinoma. Cancer 91: 2258-2262, 2001.

9. Sosa JA, Diener-West M, Gusev Y, Choti MA, Lange JR, Dooley WC, et al: Association between extent of axillary lymph node dissection and survival in patients with stage I breast cancer. Ann Surg Oncol 5: 140-149, 1998.

10. Skobe M, Hawighorst T, Jackson DG, Prevo R, Janes L, Velasco $\mathrm{P}$, et al: Induction of tumor lymphangiogenesis by VEGF-C promotes breast cancer metastasis. Nat Med 7: 192-198, 2001.

11. Stacker SA, Caesar C, Baldwin ME, Thornton GE, Williams RA, Prevo R, et al: VEGF-D promotes the metastatic spread of tumor cells via the lymphatics. Nat Med 7: 186-191, 2001.

12. Fisher B, Wolmark N, Bauer M, Redmond C and Gebhardt M: The accuracy of clinical nodal staging and of limited axillary dissection as a determinant of histologic nodal status in carcinoma of the breast. Surg Gynecol Obstet 152: 765-772, 1981.

13. De Paola F, Granato AM, Scarpi E, Monti F, Medri L, Bianchi S, et al: Vascular endothelial growth factor and prognosis in patients with node-negative breast cancer. Int J Cancer 98: 228-233, 2002.
14. Forrest AP, Stewart HJ, Roberts MM and Steele RJ: Simple mastectomy and axillary node sampling (pectoral node biopsy) in the management of primary breast cancer. Ann Surg 196: 371-378, 1982.

15. Steele RJ, Forrest AP, Gibson T, Stewart HJ and Chetty U: The efficacy of lower axillary sampling in obtaining lymph node status in breast cancer: a controlled randomized trial. Br J Surg 72: 368-369, 1985.

16. Christensen SB and Jansson C: Axillary biopsy compared with dissection in the staging of lymph nodes in operable breast cancer. A randomised trial. Eur J Surg 159: 159-162, 1993.

17. Forrest AP, Everington D, McDonald CC, Steele RJ, Chetty U and Stewart HJ: The Edinburgh randomized trial of axillary sampling or clearance after mastectomy. Br J Surg 82: 1504-1508, 1995.

18. Chetty U, Jack W, Prescott RJ, Tyler C and Rodger A: Management of the axilla in operable breast cancer treated by breast conservation: a randomized clinical trial. Edinburgh Breast Unit. Br J Surg 87: 163-169, 2000.

19. Ahlgren J, Holmberg L, Bergh J and Liljegren G: Five-node biopsy of the axilla: an alternative to axillary dissection of levels I-II in operable breast cancer. Eur J Surg Oncol 28: 97-102, 2002.

20. Cserni G: The reliability of sampling three to six nodes for staging breast cancer. J Clin Pathol 52: 681-683, 1999.

21. Cserni G: How to improve low lymph node recovery rates from axillary clearance specimens of breast cancer. A short-term audit. J Clin Pathol 51: 846-849, 1998.

22. Cserni G: Estimating the overlap between sentinel lymph nodes and axillary node samples in breast cancer. Pathol Oncol Res 5: 129-133, 1999.

23. Macmillan RD, Barbera D, Hadjiminas DJ, Rampaul RS, Lee AH, Pinder SE, et al: Sentinel node biopsy for breast cancer may have little to offer four-node-samplers. Results of a prospective comparison study. Eur J Cancer 37: 1076-1080, 2001.

24. Sato K, Tamaki K, Takeuchi H, Tsuda H, Kosuda S, Kusano S, et al: Management of the axilla in breast cancer: a comparative study between sentinel lymph node biopsy and four-node sampling procedure. Jpn J Clin Oncol 31: 318-321, 2001.

25. Yu W, Choi GS, Whang I and Suh IS: Comparison of five systems for staging lymph node metastasis in gastric cancer. Br J Surg 84: 1305-1309, 1997.

26. Inoue K, Nakane Y, Iiyama H, Sato M, Kanbara T, Nakai K, et al: The superiority of ratio-based lymph node staging in gastric carcinoma. Ann Surg Oncol 9: 27-34, 2002. 